\title{
Experimental demonstration of a novel heterogeneously integrated III-V on Si microlaser
}

\author{
Yannick De Koninck ${ }^{a}$, Fabrice Raineri ${ }^{b}$, Alexandre Bazin ${ }^{b}$, \\ Rama Raj $^{b}$, Gunther Roelkens ${ }^{a}$ and Roel Baets ${ }^{a}$ \\ ${ }^{a}$ Department of Information Technology, Photonics Research Group, Ghent University - imec \\ Center for Nano-and Biophotonics (NB-photonics) \\ Sint-Pietersnieuwstraat 41, B-9000 Ghent - Belgium \\ ${ }^{b}$ Laboratoire de Photonique et de Nanostructures, CNRS-UPR20 \\ Route de Nozay, 91460 Marcoussis, France
}

\begin{abstract}
In this work we present the first experimental demonstration of a novel class of heterogeneously integrated IIIV-on-silicon microlasers. We first show that by coupling a silicon cavity to a III-V wire, the interaction between the propagating mode in the III-V wire and the cavity mode in the silicon resonator results in high, narrow band reflection back into the III-V waveguide, forming a so-called resonant mirror. By combining two such mirrors and providing optical gain in the III-V wire in between these 2 mirrors, laser operation can be realized. We simulate the reflectivity spectrum of such a resonant mirror using 3D FDTD and discuss the results. We also present experimental results of the very first optically pumped heterogeneously integrated resonant mirror laser. The fabricated device measures $55 \mu \mathrm{m}$ by $2 \mu \mathrm{m}$ and shows single mode laser emission with a side-mode suppression ratio of $37 \mathrm{~dB}$.
\end{abstract}

Keywords: Silicon Photonics, heterogeneous integration, hybrid lasers

\section{INTRODUCTION}

Over the past decade, silicon photonics has emerged as a major technology platform for photonic integrated components and circuits. Combining the mature fabrication processes inherited from CMOS with the high refractive index contrast between the silicon waveguide core and its cladding allows for unprecedented miniaturization of passive optical components. Unfortunately, silicon has an indirect bandgap, making it extremely difficult to use it as a laser gain medium. Amongst the different solutions that have been proposed to integrate coherent light sources directly onto the silicon die, one of the most promising approaches is to bond a slab of direct band-gap material (i.e. a III-V compound) onto the silicon die. ${ }^{1}$ After bonding, the III-V substrate is removed such that only a thin film (from $<100 \mathrm{~nm}$ to a few $\mu \mathrm{m}$ thick) of III-V material remains on top of the SOI die. Now a series of lithography and etching steps are performed on the thin film to create the laser cavity. DBR- and DFB-lasers have been demonstrated ${ }^{2,3}$ on this platform where the laser mode is confined to the silicon layer and only its evanescent tail overlaps with the active III-V layer. This yields single-mode devices with high optical output, but the structures are large and require a fairly high threshold current $(>20 \mathrm{~mA})$. On the other hand, microdisk lasers were demonstrated ${ }^{1}$ with small form-factors (disk radius $<10 \mu m$ ) and low lasing threshold $(<1 \mathrm{~mA})$ but they suffer from a low side-mode suppression ratio and mode hopping. In this work, we present the very first experimental demonstration of a novel III-V on silicon microlaser that combines the best features of these two previously mentioned types of lasers. The proposed device is both small, resulting in a low threshold pump power, and offers intrinsic single-mode laser operation. Such a light source can be particularly relevant for WDM-based short-distance optical interconnects such as future inter-chip communications on a printed circuit board. Alternatively, a large number of such lasers, each lasing at a different wavelength, can be combined into an array on a single chip to obtain an efficient multi-wavelength light source that can be used in spectroscopic applications.

Further author information: (Send correspondence to Yannick De Koninck)

Yannick De Koninck: E-mail: yannick.dekoninck@intec.ugent.be, Telephone: 3292643335 


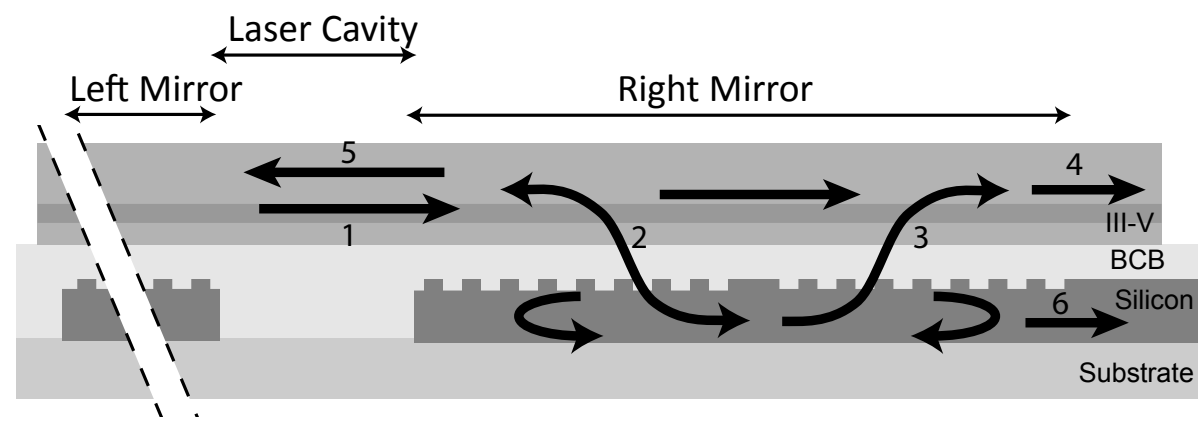

Figure 1. Schematic side-view of the operation of the resonant mirror laser.

\section{RESONANT MIRRORS}

The proposed laser structure consists of a III-V wire with two silicon cavities underneath, one under each end of the III-V wire. ${ }^{4}$ Such a silicon cavity can be a grating waveguide with a phase-shifting section in the center to create a defect-resonance. Figure 1 shows a schematic side-view of the proposed laser. Light is generated and amplified in the III-V wire where it is fully confined. As the light approaches the end of the III-V wire (1 in figure 1), a small part of the light couples to the silicon cavity underneath (2). For the wavelengths close to the silicon cavity resonance wavelength, power will start to build up inside the silicon cavity and eventually a significant amount of optical power will couple back into the III-V wire. The light coupling back co-directionally to the incident light (3) will interfere destructively to the latter, resulting in zero transmission (4). On the other hand, the light coupling back counter-directionally to the incident light (2) will provide feedback into the III-V wire (5), required to establish laser operation. Light can easily be extracted to an output waveguide by tapping a small amount of the power built-up in the silicon cavity to an external waveguide (6).

Because the power-buildup only occurs close to the silicon cavity resonance wavelength, the reflection bandwidth of this type of mirror is very narrow $(<10 \mathrm{~nm})$. Since the reflection of such a mirror can be very high and the laser mode is confined to the III- $\mathrm{V}$ wire, which maximizes the modal gain, the length of the III-V wire can be reduced to make sure only one longitudinal resonance of the III-V cavity lies within the reflection bandwidth of the mirror, yielding an intrinsically single-mode laser with a short length. Moreover, all the critical processing steps are performed in the silicon layer and no ultra-thin bonding layers are required. In fact, the design is very tolerant towards the thickness of the bonding layer, which only influences the reflection bandwidth.

\section{SIMULATION RESULTS}

The laser-structure can be split up into 3 different parts, as shown in figure 1: the left mirror, the actual laser cavity where the light is fully confined to the III-V wire and the right mirror. The propagation through the center part of the laser (the III-V wire) is straight-forward and can easily be described by considering its complex propagation constant. The interaction between the III-V waveguide and the silicon cavity in the mirror sections is much more complicated and can only be described accurately using 3D-FDTD analysis. To investigate the properties of the laser structure the analysis is split up into two different parts. First the reflectivity spectrum of the resonant mirror is calculated and optimized using 3D FDTD. Second, the properties of the full laser structure can be calculated by considering the calculated reflectivity spectrum and the mode-propagation in the III-V waveguide.

To simulate the reflectivity spectrum of the resonant mirror, the fundamental TE eigenmode of the III-V waveguide is launched into the resonant mirror structure (much like arrow $\mathbf{1}$ in figure 1). The returned light is collected (arrow 5) but also the transmitted light through the III-V wire (4) and the light coupled to the silicon waveguide $(6)$. 


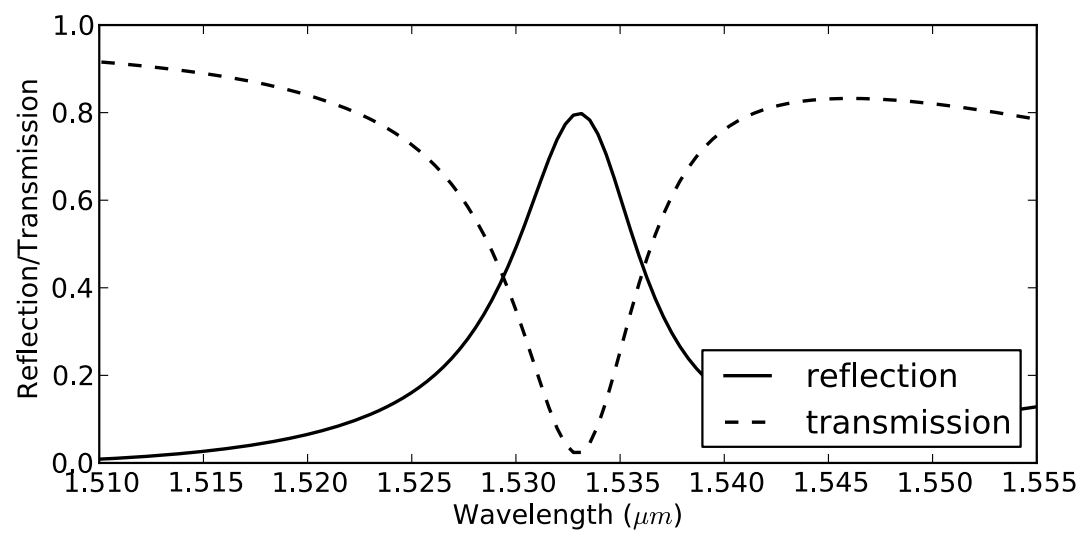

a)

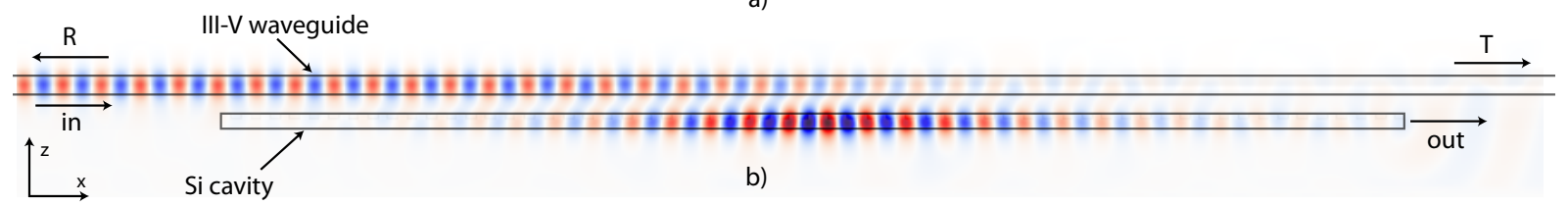

Figure 2. a) The reflection and transmission spectra of a resonant mirror laser. b) The spatial electric field distribution in a side-view cross-section at the silicon cavity's resonance wavelength.

Figure 2a depicts the simulated reflection and transmission spectrum of a resonant mirror. In this particular device, the silicon cavities are $1.6 \mu \mathrm{m}$ wide, $220 \mathrm{~nm}$ high strip waveguides with $70 \mathrm{~nm}$ deep periodic grating corrugations. Each silicon cavity consists of 60 periods ( $285 \mathrm{~nm}$ period pitch) with a quarter-wavelength defect in the middle to support a resonance near the grating's Bragg wavelength. The III-V wire above is $1.6 \mu m$ wide and consists of an $80 \mathrm{~nm}$ thick InGaAsP bulk active layer, sandwiched between two InP cladding layers. The total thickness of the III-V stack is around $250 \mathrm{~nm}$. The spacing between the silicon layer and III-V layer is $250 \mathrm{~nm}$.

The spectrum in figure 2a shows that, at the silicon cavity's resonance wavelength $(1533 \mathrm{~nm})$, the structure reflects $80 \%$ of the incoming power back into the III-V waveguide. At that same wavelength, virtually no power $(2.5 \%)$ is transmitted through the III-V waveguide. Most of the remaining $17 \%$ is directed to the output silicon waveguide. The full-width half-max bandwidth of the reflection peak amounts to $7 \mathrm{~nm}$. Figure $2 \mathrm{~b}$ shows the spatial electric field distribution in a side-view cross-section at the silicon cavity's resonance wavelength. This image clearly shows the field-enhancement in the silicon cavity due to the power build-up caused by the resonance. It also shows how the power distribution in the III-V waveguide is influenced by the presence of the silicon cavity underneath: while passing by the center of the silicon, the power in the III-V waveguide quickly decreases due to the destructive interference with the light coupling from the silicon cavity back into the III-V waveguide. This illustrates the operation of the resonant mirror described in the previous section.

The optimal distance between the two mirrors in the laser configuration is determined by the reflection bandwidth of the mirror. To guarantee single mode operation, the FSR of the laser's longitudinal modes should be at least on the order of the reflection bandwidth, so the spacing between the mirrors should be sufficiently small. On the other hand, this mirror's peak reflectivity is only $80 \%$, so the III-V waveguide should be sufficiently long to ensure that laser threshold can be reached with a reasonable pump power. Calculations show that a good value for the spacing between the silicon mirrors is $20 \mu \mathrm{m}$.

\section{DEVICE FABRICATION}

The device was fabricated starting from a SOI die processed in the $200 \mathrm{~mm}$ CMOS fab at imec - Leuven,BE and a $260 \mathrm{~nm}$ thick III-V stack on an InP substrate. The III-V stack consists of an $80 \mathrm{~nm}$ thick bulk InGaAsP 


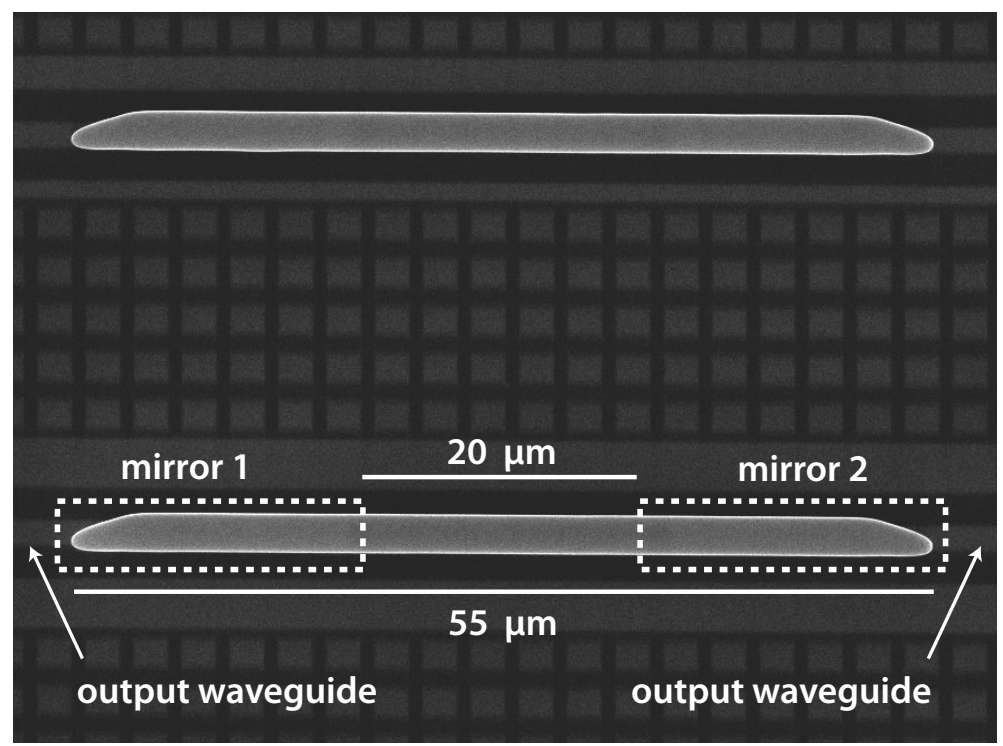

Figure 3. SEM image showing 2 finished devices. Each device is $55 \mu m$ long and about $2 \mu m$ wide.

layer sandwiched between InP cladding layers and an InGaAs etch-top layer between the laser stack and the InP substrate. The composition of the InGaAsP layer is designed to have its gain-spectrum centered around $1550 \mathrm{~nm}$. After a series of thorough cleaning procedures for both the SOI die and the III-V die, a thin layer of DVS-BCB was spin-coated on the SOI die. A $200 \mathrm{~nm}$ thick layer of $\mathrm{SiO}_{2}$ was deposited on the III-V die to increase its adhesive strength to the DVS-BCB layer and set the desired spacing between the SOI die and the III-V die. Both dies are pressed together and baked to bond the two dies together. The resulting thickness of the DVS-BCB bonding layer is around $50 \mathrm{~nm}$, yielding a total spacing between the III-V die and the SOI die of about $250 \mathrm{~nm}\left(200 \mathrm{~nm} \mathrm{SiO}_{2}+50 \mathrm{~nm}\right.$ DVS-BCB). After bonding the InP substrate is removed by first grinding off the bulk part mechanically until the thickness of the substrate has dropped to below $100 \mu \mathrm{m}$ and subsequently removing the remaining substrate in a $\mathrm{HCl}$ solution. What remains now is only a thin film (few $100 \mathrm{~nm}$ ) of III-V material bonded on top of the SOI die. After removing the etch-stop layers that prevent the $\mathrm{HCl}$ solution from etching the laser stack, a silicon nitride hard-mask is deposited. By using contact lithography the laser mesa is defined in a photo-resist layer and afterwards transferred to the silicon-nitride hard-mask layer using a plasma etch. After stripping the remaining photo-resist, the actual laser mesa is defined in the III-V film using another plasma etch with the silicon-nitride mask.

Figure 3 shows a top-view SEM image of 2 finished devices. The silicon mirror structures are masked by the III-V overlay but the white dashed boxes indicate the location and longitudinal extent of both silicon grating cavities. In this particular case, both mirrors are identical and both have a silicon output waveguide. Each laser is $55 \mu \mathrm{m}$ long and $2 \mu \mathrm{m}$ wide. The silicon cavities each consist of 60 periods with a defect (quarter-wave shift) in the center. Although devices with different grating periods were fabricated, the grating period of this particular device is $285 \mathrm{~nm}$, so each silicon cavity measures around $17 \mu \mathrm{m}$. The silicon cavities are spaced by $20 \mu \mathrm{m}$. The tapered edges of the III-V wire are intentionally patterned to avoid back-reflection from the III-V waveguide facets. Both silicon output waveguides each lead to a fiber-coupler.

\section{EXPERIMENTAL RESULTS}

The devices were measured at room temperature by illuminating each device individually from the top with an $800 \mathrm{~nm}$ DFB pump laser. This pump laser was modulated to obtain $10 \mathrm{~ns}$ pulses with $10 \mathrm{~mW}$ peak power and a repetition rate of $1.5 \mu \mathrm{s}$. Using an objective and a cylindrical lens, the shape of the illuminating spot was engineered to match the shape of the III-V waveguide to be pumped. The intensity of the pump beam is accurately controlled by a mechanized circular variable neutral density filter that is installed in the free-space 


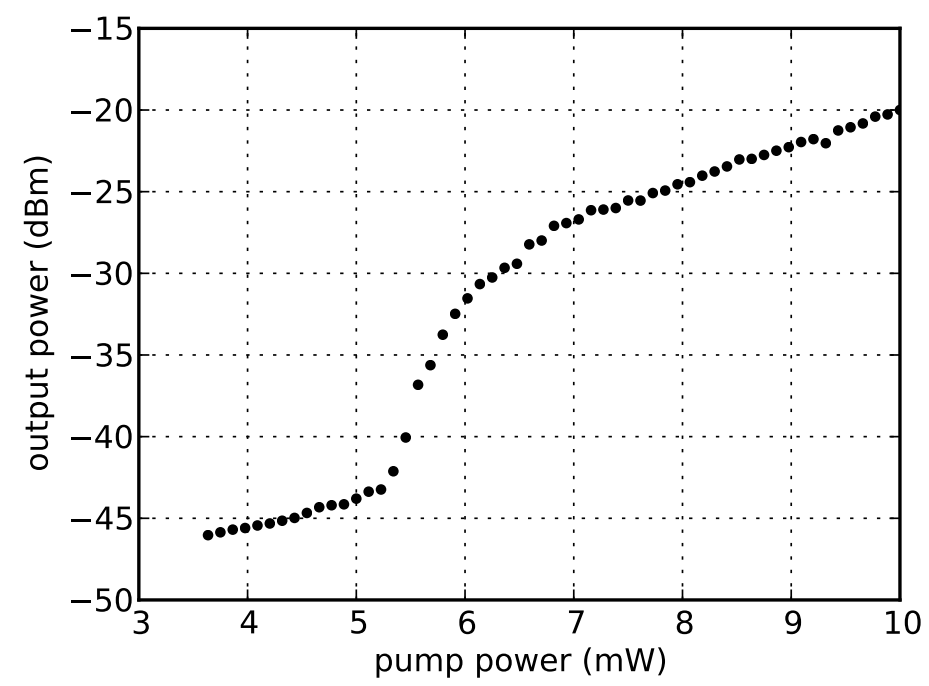

Figure 4. The laser's output power as a function of the pump power clearly showing laser threshold.

path of the pump laser leading to the device. The output of the resonant-mirror laser is coupled to a silicon waveguide which guides it towards a grating coupler. From there, a cleaved fiber directs the laser output to a spectrometer equipped with a nitrogen cooled InGaAs line camera to measure the spectral power distribution of the laser output.

To obtain the relationship between the pump power and the laser output power (the light-light curve for optically pumped devices), the output spectrum of the tested laser is measured using the spectrometer for a number of values for the pump power. Each spectrum, for the different values of the pump power, is integrated along the wavelength dimension. Because the measured output spectra are relative measurements, the values for the output-power obtained by integrating these spectra are dimensionless. By measuring the output power for one single value of the pump power using an optical power meter, it is possible to calibrate the relative power measurements. The results are shown in figure 4 . This graph very clearly shows the lasing threshold between 5 $\mathrm{mW}$ and $6 \mathrm{~mW}$ pump power. Note that the $\mathrm{x}$-axis values are the total pump laser power. Because the pumping mechanism is very inefficient, we estimate that the actual power needed to reach laser threshold is below $1 \mathrm{~mW}$. The laser peak output power is conservatively estimated to be $10 \mu \mathrm{W}$ and is limited by the available pump power.

To investigate the spectral properties of the laser, consider figure 5. This graph shows the measured output spectrum for a device with a grating period of $285 \mathrm{~nm}$. The side-mode suppression ratio is $37 \mathrm{~dB}$. More importantly, there is no evidence of spurious side-modes. The spectral longitudinal mode spacing in the cavity is estimated to be around $7 \mathrm{~nm}$, but the bandwidth of the resonant mirror is on the same order of magnitude, such that only one longitudinal mode fits within the reflection envelope of the resonant mirror and is reflected. The duration of the pump-pulse was increased and laser operation continued up until a pulse duration of $1.2 \mu s$ (with a repetition rate of $1.5 \mu \mathrm{s}$, yielding an $80 \%$ duty-cycle). The failure to operate in a continuous wave regime is most-likely due to self-heating but this can be improved in future designs by optimizing the thermal impedance of the device.

\section{CONCLUSIONS}

We present the first experimental demonstration of a hybrid III-V on silicon microlaser based on resonant mirrors. The device measures only $2 \mu m$ by $55 \mu m$ and has a high SMSR, up to $37 \mathrm{~dB}$. We believe that this concept, 


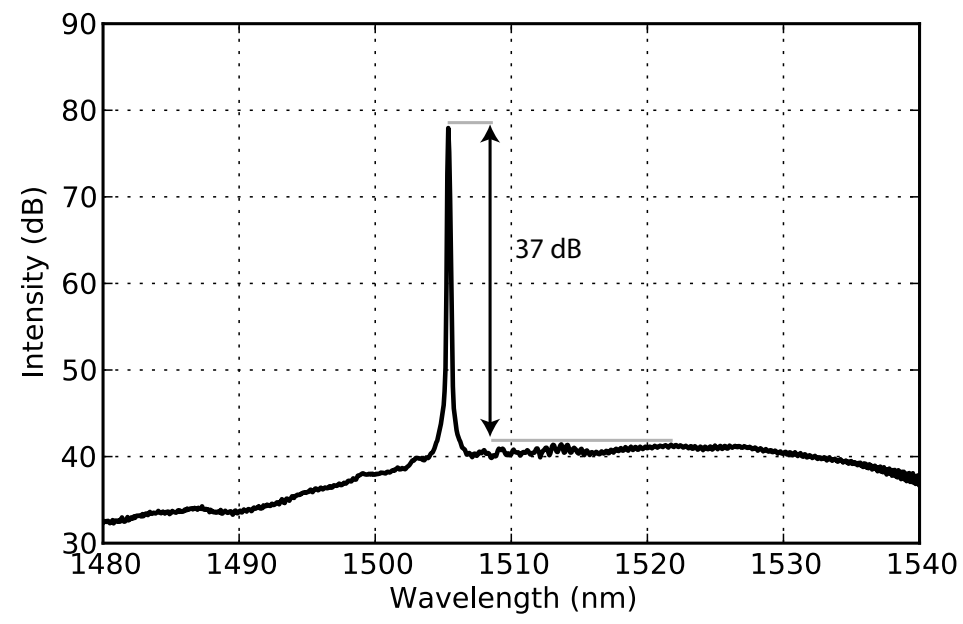

Figure 5. The measured laser output spectrum showing a side-mode suppression ratio of $37 \mathrm{~dB}$.

once electrical pumping is in place, will yield compact, single-mode hybrid microlasers with a threshold current of only a few mA's and precise control of the lasing wavelength.

\section{ACKNOWLEDGEMENTS}

The authors acknowledge partial support from IWT through the SBO-Glucosens project as well as from ERC through the InSpectra project. Yannick De Koninck thanks the research foundation Flanders (FWO) for a research grant.

\section{REFERENCES}

[1] Roelkens, G., Liu, L., Liang, D., Jones, R., Fang, A., Koch, B., and Bowers, J., "III-V/silicon photonics for on-chip and inter-chip optical interconnects," Laser $\&$ Photonics Reviews 4, 751-779 (Nov. 2010).

[2] Fang, A. W., Lively, E., Kuo, H., Liang, D., and Bowers, J. E., "A distributed feedback silicon evanescent laser," Optics Express 16, 4413-4419 (Mar. 2008).

[3] Stankovic, S., Jones, R., Sysak, M., Heck, J., Roelkens, G., and Van Thourhout, D., "Hybrid III-V/Si Distributed Feedback Laser Based on Adhesive Bonding," accepted for publication in IEEE Photonics Technology Letters .

[4] De Koninck, Y., Roelkens, G., and Baets, R., "Cavity Enhanced Reflector Based Hybrid Silicon Laser," in [IEEE Photonics Society Annual Meeting 2010], (2010). 